\title{
Rationale, Design, and Baseline Characteristics of the Utopia Trial for Preventing Diabetic Atherosclerosis Using an SGLT2 Inhibitor: A Prospective, Randomized, Open-Label, Parallel- Group Comparative Study
}

\author{
Naoto Katakami (D) Tomoya Mita - Hidenori Yoshii - Toshihiko Shiraiwa - Tetsuyuki Yasuda · Yosuke Okada • \\ Yutaka Umayahara - Hideaki Kaneto · Takeshi Osonoi - Tsunehiko Yamamoto • Nobuichi Kuribayashi • \\ Kazuhisa Maeda · Hiroki Yokoyama · Keisuke Kosugi • Kentaro Ohtoshi · Isao Hayashi • Satoru Sumitani • \\ Mamiko Tsugawa · Makoto Ohashi · Hideki Taki · Tadashi Nakamura · Satoshi Kawashima • Yasunori Sato • \\ Hirotaka Watada · Iichiro Shimomura · On behalf of the UTOPIA study investigators
}

Received: July 20, 2017 / Published online: September 1, 2017

(C) The Author(s) 2017. This article is an open access publication

\section{ABSTRACT}

Introduction: Sodium-glucose co-transporter-2 (SGLT2) inhibitors are anti-diabetic agents that improve glycemic control with a low risk of hypoglycemia and ameliorate a variety of cardiovascular risk factors. The aim of the ongoing study described herein is to investigate the

Enhanced Content To view enhanced content for this article go to http://www.medengine.com/Redeem/ D1FBF060369D16D7.

Electronic supplementary material The online version of this article (doi:10.1007/s13300-017-0292-1) contains supplementary material, which is available to authorized users.

N. Katakami $(\varangle) \cdot$ I. Shimomura

Department of Metabolic Medicine, Osaka

University Graduate School of Medicine, 2-2,

Yamadaoka, Suita, Osaka 565-0871, Japan

e-mail: katakami@endmet.med.osaka-u.ac.jp

\section{N. Katakami}

Department of Metabolism and Atherosclerosis, Osaka University Graduate School of Medicine, 2-2, Yamadaoka, Suita, Osaka 565-0871, Japan

\section{T. Mita $\cdot$ H. Watada}

Department of Metabolism and Endocrinology, Juntendo University Graduate School of Medicine, Hongo 2-1-1, Bunkyo-ku, Tokyo 113-8421, Japan preventive effects of tofogliflozin, a potent and selective SGLT2 inhibitor, on the progression of atherosclerosis in subjects with type 2 diabetes (T2DM) using carotid intima-media thickness (IMT), an established marker of cardiovascular disease (CVD), as a marker.

Methods: The Study of Using Tofogliflozin for Possible better Intervention against Atherosclerosis for type 2 diabetes patients (UTOPIA) trial is a prospective, randomized, open-label, blinded-endpoint, multicenter, and parallel-group comparative study. The aim was to recruit a total of 340 subjects with T2DM but no history of apparent CVD at 24 clinical sites and randomly allocate these to a tofogliflozin treatment group or a conventional treatment group

\author{
H. Yoshii \\ Department of Medicine, Diabetology and \\ Endocrinology, Juntendo Tokyo Koto Geriatric \\ Medical Center, Koto-ku, Tokyo 136-0075, Japan \\ T. Shiraiwa \\ Shiraiwa Medical Clinic, 4-10-24 Hozenji, Kashiwara \\ City, Osaka 582-0005, Japan \\ T. Yasuda \\ Department of Endocrinology and Metabolism, \\ Osaka Police Hospital, 10-31, Kitayama-cho, \\ Tennoji-ku, Osaka 543-0035, Japan
}


using drugs other than SGLT2 inhibitors. As primary outcomes, changes in mean and maximum IMT of the common carotid artery during a 104-week treatment period will be measured by carotid echography. Secondary outcomes include changes in glycemic control, parameters related to $\beta$-cell function and diabetic nephropathy, the occurrence of CVD and adverse events, and biochemical measurements reflecting vascular function.

Conclusion: This is the first study to address the effects of SGLT2 inhibitors on the progression of carotid IMT in subjects with T2DM without a history of CVD. The results will be available in the very near future, and these findings are expected to provide clinical data that will be helpful in the prevention of diabetic atherosclerosis and subsequent CVD.

Funding: Kowa Co., Ltd.

Clinical Trial Registration: UMIN000017607.

Keywords: Atherosclerosis;

Intima-media thickness; SGLT2 inhibitor;

Tofogliflozin

Y. Okada

First Department of Internal Medicine, School of Medicine, University of Occupational and

Environmental Health, Japan, 1-1, Iseigaoka,

Yahatanishi-ku, Kitakyushu 807-8555, Japan

Y. Umayahara

Department of Diabetes and Endocrinology, Osaka

General Medical Center, 3-1-56, Bandai-Higashi,

Sumiyoshi-ku, Osaka 558-8558, Japan

H. Kaneto

Department of Diabetes, Endocrinology and

Metabolism, Kawasaki Medical School, 577

Matsushima, Kurashiki, Okayama 701-0192, Japan

T. Osonoi

Nakakinen Clinic, 745-5, Nakadai, Naka City,

Ibaraki 311-0113, Japan

T. Yamamoto

Diabetes and Endocrinology, Kansai Rosai Hospital,

3-1-69, Inabaso, Amagasaki city, Hyogo, Japan

N. Kuribayashi

Misaki Naika Clinic, 6-44-9, Futawa-higashi,

Funabashi City, Chiba, Japan

K. Maeda

Kitasenri Maeda Clinic, 4-119, Furuedai, Suita,

Osaka 565-0874, Japan

\section{INTRODUCTION}

Atherosclerotic cardiovascular disease (CVD), which is accelerated in patients with prolonged diabetes, impairs quality of life and is a major cause of mortality $[1,2]$. Several studies have shown that abnormal glycemic metabolism plays an important role in the pathogenesis of atherosclerosis and that poor glycemic control accelerates the risk for CVD in subjects with type 2 diabetes mellitus (T2DM) [3]. However, recent studies have cast doubt on the benefits of enforcing strict glycemic control on CVD in patients with established atherosclerosis or longstanding T2DM [4-6] due to attenuation of the beneficial effects of the former by an increased incidence of hypoglycemic episodes $[7,8]$

T2DM is often closely related with insulin resistance and accompanied by other cardiovascular (CV) risk factors, such as obesity, hypertension, and dyslipidemia. Thus, early and comprehensive medical intervention to address these risk factors has been shown to be an effective strategy to prevent the development of diabetic CVD $[9,10]$. Taking into

H. Yokoyama

Jiyugaoka Medical Clinic, West 6, South 6-4-3, Obihiro, Hokkaido 080-0016, Japan

K. Kosugi

Kosugi Medical Clinic, 3-9, Tamatsukurimoto-cho, Tennoji-ku, Osaka 543-0014, Japan

K. Ohtoshi

Otoshi Medical Clinic, 8-47, Kakudacho, Osaka

Kita-ku, Osaka 530-0017, Japan

I. Hayashi

Hayashi Clinic, 3-9-23 Koshienguchi, Nishinomiya, Hyogo 663-8113, Japan

S. Sumitani

Center for Diabetes and Endocrinology, Nissay

Hospital, 6-3-8, Itachibori, Nishi-ku, Osaka

550-0012, Japan

M. Tsugawa

Department of Endocrinology and Metabolism, Ikeda Municipal Hospital, 3-1-18, Jonan, Ikeda, Osaka 563-8510, Japan

M. Ohashi

Center for Diabetes Mellitus, Osaka Rosai Hospital, 1179-3 Nagasone-cho, Kita-ku, Sakai, Osaka 591-8025, Japan 
account the multifaceted pathogenesis of CVD in diabetes, it would be an advantage to have a specific intervention which could attenuate CV risk in a multi-dimensional fashion beyond glycemic control alone.

Sodium-glucose cotransporter 2 (SGLT2) inhibitors are a new class of anti-diabetic agents that lower blood glucose concentrations by enhancing urinary glucose excretion [11]. Although they lack direct action on insulin secretion from $\beta$-cells, it is expected that their glucose-lowering effect will reduce glucotoxicity and improve $\beta$-cell function and insulin sensitivity in subjects with T2DM [12-15]. Since the mode of action of SGLT2 inhibitors is independent of insulin secretion, these agents are associated with a low risk of hypoglycemia, which is linked to an increased of CV events $[7,8,16]$. They have also been demonstrated to ameliorate a variety of $\mathrm{CV}$ risk factors and potential pathways: these agents, as monotherapy or in combination with other glucose-lowering drugs, reduce visceral adipose tissue, body weight, blood pressure, and circulating uric acid levels as well as improve serum lipid profiles [17]. These findings indicate a strong rationale for expecting that these compounds will protect against $\mathrm{CV}$ events.

Indeed, the EMPA-REG OUTCOME trial, a clinical trial designed to clarify the effects of the SGLT2 inhibitor empagliflozin on clinical CV outcomes in patients with T2DM and established CV manifestations, showed that this agent markedly reduced the risk of CV mortality

H. Taki

Diabetes Center, National Hospital Organization Osaka National Hospital, 2-1-14, Hoenzaka, Chuo-ku, Osaka 540-0006, Japan

T. Nakamura

Department of Internal Medicine, Kawasaki Hospital, 3-3-1, Higashiyamacho, Kobe Hyogo-ku, Hyogo 652-0042, Japan

S. Kawashima

Kanda Naika Clinic, 5-21-3, Hannancho, Osaka Abeno-ku, Osaka 545-0021, Japan

Y. Sato

Department of Global Clinical Research, Graduate School of Medicine, Chiba University, 1-8-1 Inohana, Chuo-ku, Chiba 260-8677, Japan compared to placebo [18]. Interestingly, however, although CV mortality and worsening heart failure were dramatically decreased, empagliflozin treatment failed to reduce macrovascular events, such as non-fatal myocardial infarction and stroke. Thus, clinical evidence regarding the therapeutic effect of SGLT2 inhibitors on atherosclerosis in patients with diabetes remains to be established.

Tofogliflozin is an SGLT2 inhibitor with 2900-fold greater selectivity for SGLT2 than SGLT1 and the highest selectivity of all clinically developed inhibitors [19]. These characteristics suggest that this agent provides efficacy with a low risk of hypoglycemia since glucose is reabsorbed by SGLT1 at a low plasma glucose level. This potent selectivity would also contribute to relatively fewer adverse events (AEs) compared to other SGLT2 inhibitors [20].

The efficacy and safety of tofogliflozin as a hypoglycemic agent have been assessed in clinical trials. A multicenter, placebo-controlled, randomized, double-blind parallel-group study involving 230 Japanese T2DM patients with inadequate glycemic control on diet/exercise therapy demonstrated that tofogliflozin 10,20 , or $40 \mathrm{mg}$ administered orally once daily for 24 weeks significantly decreased glycated hemoglobin (HbA1c), fasting blood glucose, and body weight compared to placebo [21]. A body weight reduction may be associated with a compensatory reduction of visceral fat resulting from the loss of calories following increased urinary glucose excretion [22]. Tofogliflozin also tended to reduce blood pressure to a greater extent than placebo and improved high-density lipoprotein (HDL) cholesterol levels and triglyceride levels [21]. A slight decrease in uric acid was also observed [21]. In addition, the incidence of hypoglycemia was low, and most AEs were classified as mild or moderate [21]. Another article on two phase 3 multicenter open-label randomized controlled trials in Japanese patients with T2DM also reported that tofogliflozin was well tolerated and showed sustained efficacy when patients were treated with 20 or $40 \mathrm{mg}$ tofogliflozin as monotherapy or in combination with other oral antidiabetic agents for 52 weeks [23]. These findings indicate that tofogliflozin may 
ameliorate some of the metabolic abnormalities associated with T2DM.

However, to date, no study has evaluated the long-term anti-atherosclerotic effects of tofogliflozin in patients with T2DM. Therefore, we initiated a multicenter randomized controlled trial to investigate the preventive effects of tofogliflozin on the progression of atherosclerosis in subjects with T2DM but without a history of apparent CV events, with patients not treated with tofogliflozin as the control group. In this ongoing trial, an assessment will be performed by chronologically observing the preventive effects of tofogliflozin on the progression of intima-media thickness (IMT) of the carotid artery, a surrogate endpoint of atherosclerosis [24, 25].

\section{METHODS}

\section{Study Design}

The Study of Using Tofogliflozin for Possible better Intervention against Atherosclerosis for type 2 diabetes patients (UTOPIA) trial is a prospective, randomized, open-label, blinded-endpoint, multicenter, and parallel-group comparative study. This study has been registered on the University Hospital Medical Information Network Clinical Trials Registry (UMIN-CTR), a non-profit organization in Japan, and meets the requirements of the International Committee of Medical Journal Editors (UMIN000017607).

\section{Study Population}

Japanese subjects with T2DM who periodically attended an outpatient diabetes clinic of one of 24 institutions in Japan (see Electronic Supplementary Material Table) were recruited to the study. The inclusion and exclusion criteria were as follows:

\section{Inclusion Criteria}

1. Japanese patients with T2DM and inadequate glycemic control (HbA1c $\geq 6 \%$ but $<9 \%$ ) but with an inability to achieve the blood glucose level stated in the Diabetes Treatment Guideline of 2014-2015 despite receiving medication, but not SGLT2 inhibitors, combined with diet and physical therapy, those on a specific diet and physical therapy only for at least 12 weeks, or those who received SGLT2 inhibitors in the past but who had not used SGLT2 inhibitors for at least 12 weeks before signing the consent form.

2. No changes (including new prescriptions) in their antidiabetic, antithrombotic, antihypertensive medication or a therapeutic agent for dyslipidemia for at least 12 weeks before signing their consent form.

3. Age 30-74 years at the time of giving consent.

4. Able to provide informed consent.

\section{Exclusion Criteria}

1. Type 1 or secondary diabetes.

2. In the perioperative period or with a serious infection or injury.

3. A history of myocardial infarction, angina, stroke, or cerebral infarction.

4. Severe renal dysfunction or end-stage renal failure (estimated glomerular filtration rate $<30 \mathrm{~mL} / \mathrm{min} / 1.73 \mathrm{~m}^{2}$ ).

5. Serious liver functional impairment (aspartate aminotransferase $\geq 100 \mathrm{U} / \mathrm{L}$ ).

6. Moderate to severe heart failure (class 3 or worse based on the New York Heart Association Functional Classification).

7. Urinary tract or genital infection.

8. Pregnant, possibly pregnant, nursing, or planning to conceive a child.

9. History of hypersensitivity to the study drug.

10. Present or past history of a malignant tumor (exceptions: those not on medication for malignant tumor and with no recurrence of the disease to date and without recurrence risks during this study were allowed to participate).

11. Prohibited to use tofogliflozin.

12. Other ineligibility determined by an investigator.

Screening of the study subjects was performed consecutively, and the patients who met the eligibility criteria were asked whether they 
could participate. All of the patients who agreed to participate were registered.

\section{Randomization and Study Intervention}

Patient registration occurred via the Internet through the administration office of the UTOPIA trial; once enrolled, the subjects were randomly and equally assigned to a tofogliflozin treatment group (group A) or a conventional treatment group (group B), with those in the latter group using drugs other than SGLT2 inhibitors. The randomization was performed using a dynamic allocation method based on insulin use/non-use, age, and sex.

Treatment is to be continued to achieve the target value specified in the treatment guide for diabetes [26] (generally an HbA1c of $<6.9 \%$ ) in all patients. In the conventional treatment group, either the dosage of current therapy is to be increased or a concomitant oral glucose-lowering drug (excluding the other SGLT2 inhibitors) is be added 12 weeks after randomization. In the tofogliflozin group, tofogliflozin
$20 \mathrm{mg}$ is to be administered orally once daily. However, the addition of an alternative anti-diabetic agent (excluding another SGLT2 inhibitor) is be permitted 12 weeks after randomization. In the case of hypoglycemia, the dosage of the concomitant oral glucose-lowering drug will be titrated. Anti-hyperlipidemic and anti-hypertensive drugs may be used during the study (Fig. 1).

\section{Observation Items and Schedule}

The observation items and schedule are shown in Table 1 and Fig. 1 . The study period will be 2 years after subject registration (registration period: January to November 2016; full study duration March 2015 to September 2019). All randomized participants are to be followed until the scheduled study end regardless of adherence to or discontinuation of study medication for any reason. Clinical outcomes, adherence, and AEs will be ascertained, and clinical and biochemical data are to be collected

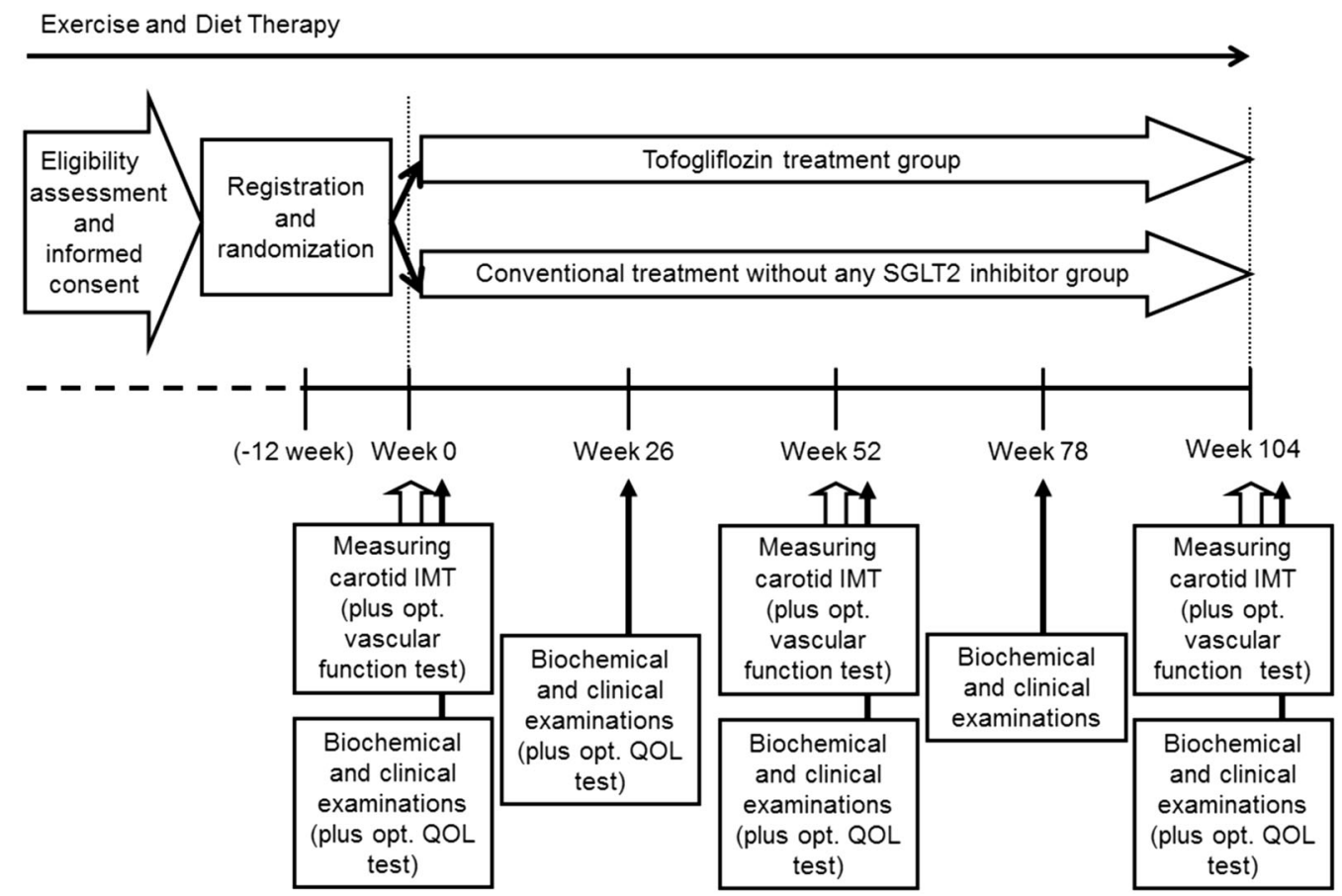

Fig. 1 Flow chart showing the study schedules. SGLT2 Sodium-glucose cotransporter 2, IMT intima-media thickness, QOL quality of life, opt. optional 
Table 1 Observation items and study schedule

\begin{tabular}{|c|c|c|c|c|c|c|c|}
\hline \multirow{3}{*}{$\begin{array}{l}\text { Observation } \\
\text { items }\end{array}$} & \multicolumn{7}{|l|}{ Schedule } \\
\hline & \multirow{2}{*}{$\begin{array}{l}\text { Registration } \\
\text { Visit } 1(-12 \\
\text { to } 0 \text { weeks }\end{array}$} & \multicolumn{5}{|c|}{ Treatment period } & \multirow{2}{*}{$\begin{array}{l}\text { At } \\
\text { discontinuation }\end{array}$} \\
\hline & & $\begin{array}{l}\text { Visit } 2 \\
\text { (0 weeks) }\end{array}$ & $\begin{array}{l}\text { Visit } 3 \\
\text { (26 weeks) }\end{array}$ & $\begin{array}{l}\text { Visit } 4 \\
\text { (52 weeks) }\end{array}$ & $\begin{array}{l}\text { Visit } 5 \\
\text { (78 weeks) }\end{array}$ & $\begin{array}{l}\text { Visit } 6 \\
\text { (104 weeks) }\end{array}$ & \\
\hline $\begin{array}{l}\text { Patient } \\
\text { characteristics }\end{array}$ & $\bigcirc$ & & & & & & \\
\hline $\begin{array}{l}\text { Body weight, } \\
\text { BMI, and AC }\end{array}$ & & $\bigcirc$ & $\bigcirc$ & $\bigcirc$ & $\bigcirc$ & $\bigcirc$ & $\bigcirc$ \\
\hline Blood pressure & & 0 & 0 & 0 & $\bigcirc$ & 0 & $\bigcirc$ \\
\hline $\begin{array}{l}\text { Blood and urine } \\
\text { tests }^{\mathrm{a}}\end{array}$ & & $\bigcirc$ & $\bigcirc$ & $\bigcirc$ & $\bigcirc$ & $\bigcirc$ & $\bigcirc$ \\
\hline $\begin{array}{l}\text { Specific blood } \\
\text { chemistry }^{\mathrm{b}}\end{array}$ & & $\bigcirc$ & & $\bigcirc$ & & 0 & $\bigcirc$ \\
\hline Carotid $\mathrm{IMT}^{\mathrm{c}}$ & & 0 & & 0 & & 0 & 0 \\
\hline $\begin{array}{l}\text { Vascular } \\
\text { function test }^{\mathrm{d}}\end{array}$ & & $\bigcirc$ & & $\bigcirc$ & & $\bigcirc$ & $\bigcirc$ \\
\hline QOL scores ${ }^{\mathrm{e}}$ & & $\bigcirc$ & $\bigcirc$ & 0 & & $\bigcirc$ & $\bigcirc$ \\
\hline Adherence & & & $\bigcirc$ & $\bigcirc$ & 0 & $\bigcirc$ & $\bigcirc$ \\
\hline Adverse events & & 0 & 0 & 0 & 0 & 0 & 0 \\
\hline
\end{tabular}

$B M I$ Body mass index, $A C$ abdominal circumference, IMT intima-media thickness, $Q O L$ quality of life

a HbA1c (glycated hemoglobin), red blood cells, white blood cells, hemoglobin, hematocrit, blood platelet count, aspartate aminotransferase, alanine aminotransferase, $\gamma$-glutamyl transferase, serum creatinine, uric acid, total cholesterol, high-density lipoprotein cholesterol, triglyceride, low-density lipoprotein cholesterol, fasting plasma glucose, amylase, spot urine, urinary microalbumin and urinary creatinine are measured

b C-peptide, fasting insulin, remnant-like particle cholesterol, high-sensitivity C-reactive protein, adiponectin, eicosapentaenoic acid/arachidonic acid ratio, $\mathrm{N}$ terminal-pro B-type natriuretic peptide, glycosylphosphatidylinositol-specific phospholipase D, T-cadherin and adiponectin-C1q complex are measured

c Mean IMT, maximum IMT, and blood vessel diameter for both sides are measured

d Optional brachial-ankle pulse wave velocity (baPWV) and ankle brachial blood pressure index (ABI) are assessed in selected sites

e Optional psychological attitudes of subjects with type 2 diabetes mellitus (T2DM) toward their diabetic therapy are quantified using the Diabetes Therapy-Related Quality of Life Questionnaire 7 (DTR-QOL7) scores

Examinations were scheduled

at $0,26,52,78$, and 104 weeks after randomization.

\section{Study Outcomes}

Primary study outcomes are the changes in mean IMT of the common carotid artery
(mean-IMT-CCA) and maximum IMT of the CCA (max-IMT-CCA) during the 104-week treatment period measured by carotid arterial echography. The change in the mean left and right mean-IMT-CCA is preliminarily defined as the most primary outcome in this study. Investigations will be performed at the start of 
the study, after 52 weeks, after 104 weeks, and at the time of any discontinuations or dosage changes.

Secondary outcomes are the changes in (1) parameters related to glycemic control and $\beta$-cell function (HbA1c, fasting plasma glucose, serum C-peptide, immunoreactive insulin, homeostasis model assessment insulin resistance (HOMA-R) and beta-cell function (HOMA)- $\beta$, and intact proinsulin/insulin ratio; (2) parameters related to diabetic nephropathy, including serum creatinine levels, urinary albumin excretion, and estimated glomerular filtration rate; (3) lipid parameters (total cholesterol, HDL cholesterol, triglyceride, low-density lipoprotein cholesterol, remnant-like particle lipoprotein, and eicosapentaenoic acid to arachidonic acid ratio); (4) biochemical measurements, including high-sensitivity C-reactive protein, adiponectin, C1q-adiponectin complex, T-cadherin, glycosyl phosphatidyl inositol-specific phospholipase $\mathrm{D}$, and $\mathrm{N}$-terminal pro-brain natriuretic peptide; (5) occurrence of $\mathrm{CV}$ events, including sudden death, ischemic heart disease, cerebral vascular disease, and peripheral artery disease; (6) any AEs; and (7) changes in treatment-related mental status. In addition, (8) subsets of consenting individuals in selected sites are enrolled in sub-studies assessing the effect of the interventions on brachial-ankle pulse wave velocity and ankle brachial blood pressure index.

\section{Safety Evaluation}

The details and incidence of adverse events are to be periodically ascertained. Based on the intention to treat the entire population, safety will be checked by recording the AEs. When the investigators confirm AEs, the severity grade, procedures, outcomes, and relationship to the study agent will be assessed. A prompt report to the study secretariat and to the data and safety monitoring board (DSMB) will then be made by the trial organizer. Any withdrawals from the study will be also reported promptly to the DSMB by the chief investigator. The DSMB will then deliberate on the incident and report the decision to the chief investigator.

\section{Measurement of Carotid IMT}

Ultrasonography scans of the carotid artery are to be performed by expert sonographers who are specifically trained to perform the prescribed study examination. To avoid inter-sonographer variability, each participant will be examined by the same sonographer with the same equipment [high-resolution B-mode ultrasound scanner equipped with a high-frequency $(>7.5-\mathrm{MHz})$ linear transducer and a limit of detection of $<0.1 \mathrm{~mm}]$ during all visits. Scanning of the extracranial CCA, the carotid bulb (Bul), and the internal carotid artery (ICA) in the neck is performed bilaterally in at least three different longitudinal projections (anterior, lateral, and posterior, which approximately correspond to $60^{\circ}, 90^{\circ}$, and $150^{\circ}$ for the right carotid artery and $210^{\circ}, 270^{\circ}$, and $300^{\circ}$ for the left carotid artery, marked on Meijer's arc) as well as in transverse projections, and the site of greatest thickness, including plaque lesions, is sought along the arterial walls. In this study, localized elevated lesions with a maximum thickness of $>1 \mathrm{~mm}$, having a point of inflection on the surface of the intima-media complex, are defined as "carotid plaque" based on the guideline of the Japan Society of Ultrasonics in Medicine [27]. The IMT is measured as the distance between two parallel echogenic lines corresponding to the vascular lumen and the adventitial layer.

To avoid inter-reader variability, all scans are to be electronically stored, sent to the central office (IMT Evaluation Committee, Osaka, Japan), and read by a single experienced reader blind to the subject's clinical characteristics in a random order using automated digital edge-detection software (Intimascope; MediaCross, Tokyo, Japan) [28]. The software system averages about 200 points of IMT values in the segment $2 \mathrm{~cm}$ proximal to the dilation of the carotid Bul (mean-IMT-CCA). In addition, the greatest thicknesses of the IMT, including plaque lesions in the CCA (max-IMT-CCA), Bul 
(max-IMT-Bul), and ICA (max-IMT-ICA), are measured separately.

\section{Sample Size}

The mean $[ \pm$ standard deviation (SD)] progression of carotid IMT in diabetic patients is considered to be $0.034 \pm 0.054 \mathrm{~mm} /$ year; a $1 \%$ improvement in the $\mathrm{HbA} 1 \mathrm{c}$ value is considered to achieves a $0.02 \mathrm{~mm} /$ year improvement in IMT [29]. Therefore, in a 2-year observation period, the registration of at least 310 patients is required to obtain $90 \%$ power to detect a difference of $0.04 \mathrm{~mm}$ in IMT between the two treatment groups, assuming an SD of $0.108 \mathrm{~mm}$ for individual differences (which was presumed to be common to both groups), a $10 \%$ dropout, and a 0.05 level of significance. The dropout rate during the 2-year observation period is assumed to be $10 \%$. According to this calculation, the target number of enrolled patients was set at 340 (170 in each group) for the 2-year registration period.

\section{Analysis Population}

All allocated participants except for those for whom IMT values are not measured at all during the observation period will be analyzed regardless of adherence using an intent-to-treat approach.

\section{Statistical Analysis}

Analyses of the primary and secondary endpoints are to be performed in the full analysis set, which will include all participants who received at least one treatment dose during the study period and who did not seriously violate the study protocol, such as by not providing informed consent or registering outside the study period. The primary analysis comparing treatment effects and the baseline-adjusted means and their 95\% confidence intervals estimated by analysis of covariance of the changes in mean and maximum IMT at 104 weeks will be compared between the treatment groups. The results will be adjusted by allocation factors. The primary analysis will not include missing observations, with the mixed-effects model for repeated measures (MMRM) being used as a sensitivity analysis to examine the effect of the missing data. In addition, MMRM will be used as a sensitivity analysis to examine the outcomes at baseline and 104 weeks modeled as a function of time, treatment, and treatment-by-time interactions. The secondary analysis will be performed in the same manner as the primary analysis.

All comparisons are planned and all $p$ values will be two-sided. $P$ values of $<0.05$ will be considered to be statistically significant. All statistical analyses will be performed using SAS software version 9.4 (SAS Institute, Cary, NC). The statistical analysis plan will be developed by the principal investigator and a biostatistician before the completion of patient recruitment and database locking.

\section{Compliance with Ethics Guidelines}

The protocol was approved by the institutional review board at each participating institution in compliance with the Declaration of Helsinki and current legal regulations in Japan. The study will be conducted in accordance with the Ethical Guidelines for Medical and Health Research Involving Human Subjects published by the Ministry of Health, Labour and Welfare of Japan and the Helsinki Declaration of 1964, as revised in 2013. Written informed consent for participation and publication was obtained from all the participants after a full explanation of the study was provided.

\section{Trial Organization}

The UTOPIA trial was designed by the principal investigators (Iichiro Shimomura, Osaka University Graduate School of Medicine, Osaka, Japan and Hirotaka Watada, Juntendo University School of Medicine, Tokyo, Japan) through the UTOPIA Project Office based at the Department of Metabolic Medicine, Osaka University Graduate School of Medicine, Osaka, Japan. The principal investigators are responsible for all aspects of trial management, including collecting and cleaning all data, handling all 
protocol-related issues, monitoring and optimizing adherence to interventions, adjudicating outcomes, auditing the study progress, and determining, executing, and publishing the final study analysis. We also have an IMT Evaluation Committee and a Clinical Events Committee, which are independent of this trial; the former is as described in the "Measurement of Carotid IMT" section, while the latter is composed of two cardiologists and one neurologist.

\section{RESULTS}

Between January 2016 and November 2016, 340 participants were recruited at 24 clinical sites and randomly allocated either to tofogliflozin treatment group (group A, 169 patients) or a conventional treatment group (group B, 171 patients) using drugs other than SGLT2 inhibitors.

\section{CONCLUSION}

Many clinical studies have shown that HbA1c level is associated with the risk of CVD in patients with T2DM [3]; consequently, the management of glucose levels represents one of the principal treatment goals of diabetes therapy. However, despite the legacy effect of long-term intensive glycemic intervention [30-32], recent studies have cast doubt on the benefits of strict glycemic control on CVD in patients with established atherosclerosis or longstanding T2DM [4-6], with attenuation of the beneficial effects of glycemic control likely due to the increased incidence of hypoglycemic episodes $[7,8]$. In addition, since early and comprehensive medical intervention for the accompanying $\mathrm{CV}$ risk factors, such as obesity, hypertension, and dyslipidemia, has been shown to effectively prevent the development of diabetic CVD $[9,10]$, early medical intervention using a comprehensive approach beyond glycemic control alone would be an advantage.

SGLT2 inhibitors are oral anti-diabetic agents that have the potential to improve glycemic control with a low risk of hypoglycemia. This class of agents has also been demonstrated to ameliorate a variety of $\mathrm{CV}$ risk factors via its metabolic, hemodynamic, renal, and neurohormonal effects [17, 33]. In addition, an increase in blood ketone bodies is frequently detected in patients receiving SGLT2 inhibitor treatment, considered to be due to an increase in lipolysis and mobilization of lipids/free fatty acids to compensate for glucose loss via the urine. Although the effect of such an increase in blood ketone bodies on safety is unknown, the ketone metabolite $\beta$-hydroxybutyrate may prevent the progression of atherosclerosis by blocking danger signaling in vascular cells. The activation of the NOD-like receptor family pyrin domain-containing 3 (NLRP3) inflammasome is required for caspase- 1 to convert inactive pro-interleukin (IL)-1beta and pro-IL-18 into their bioactive and secreted forms as crucial mediators of inflammation in the vessel wall [34]. It has recently been reported that $\beta$-hydroxybutyrate blocks NLRP3 inflammasome-mediated inflammation [35]. Considering the pleiotropic anti-atherogenic effect of SGLT2 inhibitors mentioned above, they are expected to inhibit the progression of atherosclerosis.

We projected their results of several clinical CV outcome trials of SGLT2 inhibitors in designing our study to address the clinically important question of whether SGLT2 inhibitors can accomplish the elusive goal of an anti-diabetic agent, such as reducing the incidence of CVD [18, 36]. Among these, the results of the EMPA-REG OUTCOME trial, which was designed to clarify the effects of the SGLT2 inhibitor empagliflozin on clinical CV safety in patients with T2DM and established CV manifestations, have been published. This trial showed that empagliflozin markedly reduced the risk of $\mathrm{CV}$ mortality compared to placebo, but there was no significant risk reduction of macrovascular complications, such as non-fatal myocardial infarction and stroke, suggesting that possible mechanisms may be largely hemodynamic effects induced by glycosuria and natriuresis rather than a direct anti-atherothrombotic effect [18, 37-39]. Thus, at this point in time, no study has demonstrated that SGLT2 inhibitors prevent the development of atherosclerosis in patients with T2DM. In addition, to the best of our knowledge, very few 
clinical trials have investigated whether SGLT2 inhibitors can prevent the development of atherosclerosis in subjects with T2DM but no apparent CVD.

The UTOPIA trial is a multicenter, prospective, randomized, investigator-initiated clinical trial with the aim to investigate the preventive effects of tofogliflozin, a potent and selective SGLT2 inhibitor, on the progression of atherosclerosis in subjects with T2DM but without a history of apparent atherosclerotic disease, using common carotid IMT, a widely used surrogate marker of atherosclerosis, as the primary endpoint. We use surrogate endpoints for this trial due to many practical constraints, including trial costs and concern regarding the feasibility of performing long-term intervention. Carotid ultrasound measurements of IMT have been validated against pathologic specimens and demonstrated to be strong predictors of $\mathrm{CV}$ events in subjects with and without T2DM [40, 41]. It has also been shown that changes in carotid IMT over time correlate with rates of future CV events [42]. Therefore, several clinical studies have assessed the inhibitory effects of a variety class of anti-diabetic agents (e.g. biguanides, thiazolidinediones, alpha-glucosidase inhibitors, and DPP-4 inhibitors) on carotid IMT progression [43-48]. Although the UTOPIA study has a multicenter open-label design, repeated IMT measurements are planned in a blinded manner, and the analyses will be performed at a core laboratory to avoid bias and measurement errors between institutions: all scans will be electronically stored and sent to the core laboratory (IMT Evaluation Committee, Osaka, Japan) and read by a single experienced reader unaware of the patients' clinical characteristics, in a random order using automated digital edge-detection software (Intimascope; MediaCross) [28]. The same systematic procedures for analyzing carotid IMT were used in our previous studies $[47,48]$. The reliability and reproducibility of measurements of carotid IMT has been certified in the current study.

To the best of our knowledge, no study to date has investigated the effects of SGLT2 inhibitors on changes in carotid IMT in diabetic subjects. Tanaka et al. recently published the protocol of the PROTECT study [49], the only ongoing study to assess the preventive effect of SGLT2 inhibitors on the progression of carotid atherosclerosis using carotid IMT as a surrogate marker. The PROTECT study is a 24-month prospective study in 480 Japanese subjects with T2DM, with the aim to assess the preventive effect of ipragliflozin, another SGLT2 inhibitor, on the progression of carotid IMT. Although the UTOPIA study design looks much like that of the PROTECT study, there are several differences. First, the SGLT2 inhibitor used as test drug in each study is different, with tofogliflozin to be tested in the UTOPIA study but ipragliflozin in the PROTECT study. Next, there are several differences in the eligibility criteria between two studies, which could lead to the differences in the clinical characteristics of the study subjects. In particular, the UTOPIA study excludes patients with a history of CVD, while the PROTECT study does not exclude patients with a history of CVD, although patients with a history of coronary artery disease, coronary vascularization, open-heart surgery, stroke, or transient ischemic attack $\leq 3$ months prior to eligibility are excluded.

Several limitations of our study should be discussed. First, it is not a double-blind, placebo-controlled trial but rather a prospective, randomized, open blinded, endpoint study. Although the determination of endpoints is blinded and conducted by expert committees, medications are open-label, which may still induce unexpected bias. Second, it is possible that the additional anti-diabetic agents administered when glycemic control becomes worse, especially in the control group, may influence the outcomes. Previous studies have shown that several classes of hypoglycemic agents, including DDP-4 inhibitors, suppress the progression of carotid IMT [43-48]. Third, the subjects of this study are Japanese patients with T2DM, a cohort with a relatively low CV risk. Therefore, it would be premature to generalize our findings to other racial or ethnic groups. It should be also noted that anti-diabetic agent prescribing in patients with T2DM in Japan is somewhat different from that in Western countries. In treatment algorithms of T2DM in Western countries, biguanides are recommended as first-line agents. In contrast, 
the treatment guideline of the Japan Diabetes Society does not specify first-line drugs and leaves the selection of anti-diabetic agents to the treating physicians. The guideline recommends selecting "glucose-lowering agents based on the disease condition of each particular patient with consideration given to the pharmacological and safety profile of each glucose-lowering agent".

Notwithstanding these limitations, our study is expected to provide clinical data that will be helpful in the prevention of diabetic atherosclerosis and subsequent cardiovascular and cerebrovascular disease. This is a very important objective because the worldwide incidences of diabetes and diabetic atherosclerosis are increasing at staggering rates, and the economic and social costs to the individuals and countries will grow enormously in the near future. Results from this important clinical trial are planned to be published in 2019 .

\section{ACKNOWLEDGEMENTS}

The authors thank all of the staff and patient participants of this study. The authors gratefully acknowledge the assistance of H Yamada (Soiken Holdings Inc., Tokyo Japan) and Editage by Cactus Communications Inc. (Tokyo Japan) for providing editorial assistance and proofreading the manuscript. This assistance was funded by Kowa Co., Ltd., Tokyo, Japan.

Funding information. Financial support for this study including journal's article processing charges was provided by Kowa Co., Ltd., Tokyo, Japan.

Authors' contributions. All named authors meet the International Committee of Medical Journal Editors (ICMJE) criteria for authorship for this manuscript. All authors had full access to all of the data in this study and take complete responsibility for the integrity of the data and accuracy of the data analysis, as well as for the integrity of the work as a whole. All authors have given final approval to the version to be published.
Disclosures. Naoto Katakami is a staff member of the endowed chair (Department of Metabolism and Atherosclerosis) established by funds from Kowa Co., Ltd., and has received research funds from MSD and lecture fees from Astellas Pharma Inc., AstraZeneca K.K., Boehringer Ingelheim, Daiichi Sankyo Inc., Dainippon Sumitomo Pharma Co., Eisai Co., Eli Lilly, Kowa Pharmaceutical Co., Kyowa Hakko Kirin Co. Ltd., Mitsubishi Tanabe Pharma Co., Novartis Pharmaceuticals, Novo Nordisk Pharma, Ono Pharmaceutical Co., Takeda Pharmaceutical Co., Sanofi-Aventis, and Shionogi \& Co. Tomoya Mita has received lecture fees from Ono Pharmaceutical Co., Ltd., Takeda Pharmaceutical Company Limited, scholarship donations from MSD K.K., Astellas Pharma Inc., AstraZeneca K.K., Ono Pharmaceutical Co., Ltd., Kyowa Hakko Kirin Co. Ltd., Sanofi-Aventis K.K., Daiichi Sankyo Company, Limited, Sumitomo Dainippon Pharma Co., Ltd., Takeda Pharmaceutical Company Limited, Mitsubishi Tanabe Pharma Corporation, Terumo Corporation, Nippon Boehringer Ingelheim Co., Ltd., Novo Nordisk Pharma Ltd., Pfizer Japan Inc., Benefit One Health Care Inc., Mochida Pharmaceutical Co., Ltd., and Nitto Boseki Co., Ltd. as well as funds of endowed chair from MSD K.K., Takeda Pharmaceutical Company Limited. Toshihiko Shiraiwa has received lecture fees from Sanofi-Aventis K.K. and Takeda Pharmaceutical Company Limited and research funding from Novo Nordisk Pharma Ltd., Sanofi-Aventis K.K., Takeda Pharmaceutical Company Limited, AstraZeneca K.K., Nippon Boehringer Ingelheim Co., Ltd., and Mitsubishi Tanabe Pharma Corporation. Tetsuyuki Yasuda has received lecture fees from Nippon Boehringer Ingelheim Co., Ltd. and Sanofi-Aventis K.K. Yosuke Okada has received lecture fees from Astellas Pharma Inc., MSD K.K., Ono Pharmaceutical Co., Ltd., Mitsubishi Tanabe Pharma Corporation, Bayer Holding Ltd., Novartis Pharmaceuticals Corp., Novo Nordisk Pharma Ltd., Eli Lilly Japan K.K., and Kissei Pharmaceutical Co., Ltd. as well as research funding from Kowa Pharmaceutical Co. Ltd. and Mitsubishi Tanabe Pharma Corporation. Hideaki Kaneto has received lecture fees from Nippon Boehringer Ingelheim Co., Ltd., Sanofi-Aventis 
K.K., Novo Nordisk Pharma Ltd., Eli Lilly Japan K.K., MSD K.K., Ono Pharmaceutical Co., Ltd., Takeda Pharmaceutical Company Limited, Daiichi Sankyo Company, Limited, Mitsubishi Tanabe Pharma Corporation, AstraZeneca K.K., Astellas Pharma Inc., Novartis Pharmaceuticals Corp., and Sumitomo Dainippon Pharma Co.; scholarship donations from Novo Nordisk Pharma Ltd., Nippon Boehringer Ingelheim Co., Ltd., Sumitomo Dainippon Pharma Co., Ltd., Ono Pharmaceutical Co., Ltd., Sanofi-Aventis K.K., Eli Lilly Japan K.K., Astellas Pharma Inc., Daiichi Sankyo Company, Limited, Mitsubishi Tanabe Pharma Corporation, MSD K.K., Takeda Pharmaceutical Company Limited, AstraZeneca K.K., Mochida Pharmaceutical Co., Ltd., Taisho Toyama Pharmaceutical Co., Kissei Pharmaceutical Co., Ltd., and Kyowa Hakko Kirin Co. Ltd. Takeshi Osonoi has received lecture fees from Takeda Pharmaceutical Company Limited, Astellas Pharma Inc., Novo Nordisk Pharma Ltd., and Sanwa Kagaku Kenkyusho Co., Ltd.; manuscript fees from Sanwa Kagaku Kenkyusho Co., Ltd.; and research funding from Takeda Pharmaceutical Company Limited, Novo Nordisk Pharma Ltd., Astellas Pharma Inc., Sanwa Kagaku Kenkyusho Co., Ltd., Mitsubishi Tanabe Pharma Corporation, Nippon Boehringer Ingelheim Co., Ltd., Taisho Pharmaceutical Co., Ltd., Eli Lilly Japan K.K., Daiichi Sankyo Company, Limited, Bayer Holding Ltd., Kowa Pharmaceutical Co. Ltd., and AbbVie Inc. Nobuichi Kuribayashi has received lecture fees from Takeda Pharmaceutical Company Limited, Sanofi-Aventis K.K., Novo Nordisk Pharma Ltd., MSD K.K., and Mitsubishi Tanabe Pharma Corporation. Satoru Sumitani has received lecture fees from Sumitomo Dainippon Pharma Co., Ltd. Yasunori Sato has received lecture fees from Siemens K.K. Hirotaka Watada has received lecture fees from Sanofi-Aventis K.K., MSD K.K., Astellas Pharma Inc., Takeda Pharmaceutical Company Limited, Mitsubishi Tanabe Pharma Corporation, AstraZeneca K.K., Nippon Boehringer Ingelheim Co., Ltd., Eli Lilly Japan K.K., Novo Nordisk Pharma Ltd., Sanwa Kagaku Kenkyusho Co., Ltd., Kowa Co., Ltd., Novartis Pharmaceuticals Corp., Daiichi Sankyo Company, Limited, Kyowa Hakko Kirin Co. Ltd., Ono Pharmaceutical Co., Ltd., and Kissei
Pharmaceutical Co., Ltd.; research funding from Novartis Pharmaceuticals Corp., Eli Lilly Japan K.K., scholarship donations from MSD K.K., Astellas Pharma Inc., AstraZeneca K.K., Abbot Japan Co., Ltd., Ono Pharmaceutical Co., Ltd., Kissei Pharmaceutical Co., Ltd., Kyowa Hakko Kirin Co. Ltd., Kowa Pharmaceutical Co. Ltd., Sanofi-Aventis K.K., Sanwa Kagaku Kenkyusho Co., Ltd., Johnson \& Johnson K.K., Daiichi Sankyo Company, Limited, Sumitomo Dainippon Pharma Co., Ltd., Terumo Corporation, Taisho Toyama Pharmaceutical Co., Ltd., Takeda Pharmaceutical Company Limited, Mitsubishi Tanabe Pharma Corporation, Nitto Boseki Co., Ltd., Nippon Boehringer Ingelheim Co., Ltd., Novartis Pharmaceuticals Corp., Novo Nordisk Pharma Ltd., Bayer Holding Ltd., Pfizer Japan Inc., Bristol-Myers Squibb K.K., Benefit one Health care Inc., and Mochida Pharmaceutical Co., Ltd.; and funds of endowed chair from MSD K.K. and Takeda Pharmaceutical Company Limited. Iichiro Shimomura has received lecture fees from Astellas Pharma Inc., AstraZeneca K.K., MSD K.K., Ono Pharmaceutical Co., Kyowa Hakko Kirin Co., Kowa Pharmaceutical Co., Sanofi K.K., Sanwa Kagaku Kenkyusho Co., Daiichi Sankyo Co., Takeda Pharma K.K., Mitsubishi Tanabe Pharma Co., Teijin Pharma, Eli Lilly Japan K.K., Nippon Boehringer Ingelheim Co., Novartis Pharma K.K., Novo Nordisk Pharma, Bayer Yakuhin, Pfizer Japan Inc., Bristol-Myers K.K., Mochida Pharmaceutical Co., Shionogi \& Co., Taisho Toyama Pharmaceutical Co., and Shionogi \& Co.; and research funds from Astellas Pharma Inc., AstraZeneca K.K., Eisai Co., MSD K.K, Otsuka Pharmaceutical Co., Ono Pharmaceutical Co., Kaken Pharmaceutical Co., Kissei Pharmaceutical Co., Kyowa Hakko Kirin Co., Sanofi K.K., Shionogi \& Co., Daiichi Sankyo Co., Dainippon Sumitomo Pharma Co., Takeda Pharma K.K., Mitsubishi Tanabe Pharma Co., Teijin Pharma, Nippon Boehringer Ingelheim Co., Novartis Pharma K.K., Novo Nordisk Pharma, Pfizer Japan Inc., Bristol-Myers K.K., Mochida Pharmaceutical Co., Eli Lilly Japan K.K, Kowa Co., Ltd., Kowa Pharmaceutical Co., and Taisho Toyama Pharmaceutical Co. Hidenori Yoshii, Yutaka Umayahara, Tsunehiko Yamamoto, Kazuhisa Maeda, Hiroki Yokoyama, Keisuke Kosugi, Kentaro Ohtoshi, Isao Hayashi, 
Mamiko Tsugawa, Makoto Ohashi, Hideki Taki, Tadashi Nakamura, and Satoshi Kawashima declare that they have no conflict of interest.

Compliance with Ethical Guidelines. All procedures followed were in accordance with the ethical standards of the responsible committee on human experimentation (institutional and national) and with the Helsinki Declaration of 1964, as revised in 2013. Informed consent was obtained from all patients for being included in the study.

Data Availability. The datasets generated during and/or analyzed during the current study will be available from the corresponding author on reasonable request.

Open Access. This article is distributed under the terms of the Creative Commons Attribution-NonCommercial 4.0 International License (http://creativecommons.org/licenses/ by-nc/4.0/), which permits any noncommercial use, distribution, and reproduction in any medium, provided you give appropriate credit to the original author(s) and the source, provide a link to the Creative Commons license, and indicate if changes were made.

\section{REFERENCES}

1. Haffner SM, Lehto S, Rönnemaa T, Pyörälä $K$, Laakso M. Mortality from coronary heart disease in subjects with type 2 diabetes and in nondiabetic subjects with and without prior myocardial infarction. N Engl J Med. 1998;339:229-34.

2. Stamler J, Vaccaro O, Neaton JD, Wentworth D. Diabetes, other risk factors, and 12-year cardiovascular mortality for men screened in the multiple risk factor interventional trial. Diabetes Care. 1993;16:434-44.

3. Laakso M. Glycemic control and the risk for coronary heart disease in patients with non-insulin-dependent diabetes mellitus. The finnish studies. Ann Intern Med. 1996;124:127-30.

4. Patel A, MacMahon S, Chalmers J, Neal B, Billot L, Woodward $\mathrm{M}$, et al. Intensive blood glucose control and vascular outcomes in patients with type 2 diabetes. N Engl J Med. 2008;358:2560-72.
5. Duckworth W, Abraira C, Moritz T, Reda D, Emanuele N, Reaven PD, et al. Glucose control and vascular complications in veterans with type 2 diabetes. N Engl J Med. 2009;360:129-39.

6. Gerstein HC, Miller ME, Byington RP, Goff DC Jr, Bigger JT, Buse JB, et al. Effects of intensive glucose lowering in type 2 diabetes. $\mathrm{N}$ Engl J Med. 2008;358:2545-59.

7. Frier BM, Schernthaner G, Heller SR. Hypoglycemia and cardiovascular risks. Diabetes Care. 2011;34[Suppl 2]:S132-7.

8. Khunti K, Davies M, Majeed A, Thorsted BL, Wolden ML, Paul SK. Hypoglycemia and risk of cardiovascular disease and all-cause mortality in insulin-treated people with type 1 and type 2 diabetes: a cohort study. Diabetes Care. 2015;38:316-22.

9. Gaede P, Lund-Andersen H, Parving HH, Pedersen O. Effect of a multifactorial intervention on mortality in type 2 diabetes. $\mathrm{N}$ Engl J Med. 2008;358:580-91.

10. Dailey G. Early and intensive therapy for management of hyperglycemia and cardiovascular risk factors in patients with type 2 diabetes. Clin Ther. 2011;33:665-78.

11. Gerich JE. Role of the kidney in normal glucose homeostasis and in the hyperglycaemia of diabetes mellitus: therapeutic implications. Diabet Med. 2010;27:136-42.

12. Jurczak MJ, Lee HY, Birkenfeld AL, Jornayvaz FR, Frederick DW, Ponqratz RL, Zhao X. SGLT2 deletion improves glucose homeostasis and preserves pancreatic beta-cell function. Diabetes. 2011;60:890-8.

13. Ferrannini E, Muscelli E, Frascerra S, Baldi S, Mari A, et al. Metabolic response to sodium-glucose cotransporter 2 inhibition in type 2 diabetic patients. J Clin Invest. 2014;124:499-508.

14. Merovci A, Solis-Herrera C, Daniele G, Eldor R, Fiorentino TV, et al. Dapagliflozin improves muscle insulin sensitivity but enhances endogenous glucose production. J Clin Invest. 2014;124:509-14.

15. Takahara M, Shiraiwa T, Matsuoka TA, Katakami N, Shimomura I. Ameliorated pancreatic $\beta$ cell dysfunction in type 2 diabetic patients treated with a sodium-glucose cotransporter 2 inhibitor ipragliflozin. Endocr J. 2015;62:77-86.

16. Hsu PF, Sung SH, Cheng HM, Yeh JS, Liu WL, Chan $\mathrm{WL}$, et al. Association of clinical symptomatic hypoglycemia with cardiovascular events and total mortality in type 2 diabetes. Diabetes Care. 2013;36:894-900. 
17. Vasilakou D, Karagiannis T, Athanasiadou E, et al. Sodium glucose cotransporter 2 inhibitors for type 2 diabetes: a systematic review and meta-analysis. Ann Intern Med. 2013;159:262-74.

18. Zinman B, Wanner C, Lachin JM, Fitchett D, Bluhmki E, Hantel S, et al. Empagliflozin, cardiovascular outcomes, and mortality in type 2 diabetes. N Engl J Med. 2015;373:2117-28.

19. Suzuki M, Honda K, Fukazawa M, Ozawa K, Hagita $\mathrm{H}$, Kawai T, et al. Tofogliflozin, a potent and highly specific sodium/glucose cotransporter 2 inhibitor, improves glycemic control in diabetic rats and mice. J Pharmacol Exp Ther. 2012;341:692-701.

20. Nagata T, Fukazawa M, Honda K, Yata T, Kawai M, Yamane M, et al. Selective SGLT2 inhibition by tofogliflozin reduces renal glucose reabsorption under hyperglycemic but not under hypo-or euglycemic conditions in rats. Am J Physiol Endocrinol Metab. 2013;304:E414-23.

21. Kaku K, Watada H, Iwamoto Y, Utsunomiya K, Terauchi Y, Tobe K, for The Tofogliflozin 003 Study Group, et al. Efficacy and safety of monotherapy with the novel sodium/glucose cotransporter-2 inhibitor tofogliflozin in Japanese patients with type 2 diabetes mellitus: a combined Phase 2 and 3 randomized, placebo-controlled, double-blind, parallel-group comparative study. Cardiovasc Diabetol. 2014;13:65.

22. Kleta R, Stuart C, Gill FA, Gahl WA. Renal glucosuria due to SGLT2 mutations. Mol Genet Metab. 2004;82:56-8.

23. Tanizawa Y, Kaku K, Araki E, Tobe K, Terauchi Y, Utsunomiya K, for the Tofogliflozin 004 and 005 Study group, et al. Long-term safety and efficacy of tofogliflozin, a selective inhibitor of sodium-glucose cotransporter 2, as monotherapy or in combination with other oral antidiabetic agents in Japanese patients with type 2 diabetes mellitus: multicenter, open-label, randomized controlled trials. Expert Opin Pharmacother. 2014;15:749-66.

24. Yamasaki Y, Kodama M, Nishizawa H, Sakamoto K, Matsuhisa M, Kajimoto Y, et al. Carotid intimamedia thickness in Japanese type 2 diabetic subjects: predictors of progression and relationship with incident coronary heart disease. Diabetes Care. 2000;23:1310-5.

25. Mitsuhashi N, Onuma T, Kubo S, Takayanagi N, Honda M, Kawamori R. Coronary artery disease and carotid artery intima-media thickness in Japanese type 2 diabetic patients. Diabetes Care. 2002;25:1308-12.

26. The Japan Diabetes Society. Treatment guide for diabetes 2014-2015, Editorial Committee members of "Treatment guide for diabetes" (Araki E, Inagaki $\mathrm{N}$, Inoguchi $\mathrm{T}$, Utsunomiya $\mathrm{K}$, Tanizawa $\mathrm{Y}$, Nakamura J, Noda M, Watada H, Imamura S), editors. Tokyo: Bunkodo Co., Ltd., 2014.

27. Terminology and Diagnostic Criteria Committee, Japan Society of Ultrasonics in Medicine. Subcommittee for preparing guidelines for ultrasound diagnosis of carotid artery: standard method for ultrasound evaluation of carotid artery lesions. Jpn J Med Ultrasonics. 2009;36:501-18.

28. Yanase T, Nasu S, Mukuta Y, Shimizu Y, Nishihara $\mathrm{T}$, Okabe $\mathrm{T}$, et al. Evaluation of a new carotid intima-media thickness measurement by B-mode ultrasonography using an innovative measurement software, Intimascope. Am J Hypertens. 2006;19:1206-12.

29. Yokoyama H, Katakami N, Yamasaki Y. Recent advances of intervention to inhibit progression of carotid intima-media thickness in patients with type 2 diabetes mellitus. Stroke. 2006;37:2420-7.

30. Skyler JS, Bergenstal R, Bonow RO, Buse J, Deedwania $\mathrm{P}$, Gale EA, et al. Intensive glycemic control and the prevention of cardiovascular events: implications of the ACCORD, ADVANCE, and VA diabetes trials: a position statement of the American Diabetes Association and a scientific statement of the American College of Cardiology Foundation and the American Heart Association. Diabetes Care. 2009;32:187-92.

31. Zarich SW. Antidiabetic agents and cardiovascular risk in type 2 diabetes. Nat Rev Endocrinol. 2009;5:500-6.

32. Holman RR, Paul SK, Bethel MA, Matthews DR, Neil HA. 10-year follow-up of intensive glucose control in type 2 diabetes. N Engl J Med. 2008;359:1577-89.

33. Inzucchi SE, Zinman B, Wanner C, Ferrari $\mathrm{R}$, Fitchett D, Hantel S, et al. SGLT-2 inhibitors and cardiovascular risk: proposed pathways and review of ongoing outcome trials. Diabetes Vasc Dis Res. 2015;12:90-100.

34. Zimmer S, Grebe A, Latz E. Danger signaling in atherosclerosis. Circ Res. 2015;116:323-40.

35. Youm YH, Nguyen KY, Grant RW, Goldberg EL, Bodogai M, Kim D, et al. The ketone metabolite $\beta$-hydroxybutyrate blocks NLRP3 inflammasome-mediated inflammatory disease. Nat Med. 2015;21:263-9.

36. Neal B, Perkovic V, de Zeeuw D, Mahaffey KW, Fulcher G, Stein P, et al. Rationale, design, and baseline characteristics of the canagliflozin cardiovascular assessment study (CANVAS) - a randomized placebo-controlled trial. Am Heart J. 2013;166:217-223.e11. 
37. Sattar N, McLaren J, Kristensen SL, Preiss D, McMurray JJ. SGLT2 inhibition and cardiovascular events: why did EMPA-REG outcomes surprise and what were the likely mechanisms? Diabetologia. 2016;59:1333-9.

38. Abdul-Ghani M, Del Prato S, Chilton R, DeFronzo RA. SGLT2 inhibitors and cardiovascular risk: lessons learned from the EMPA-REG OUTCOME study. Diabetes Care. 2016;39:717-25.

39. Heerspink HJ, Perkins BA, Fitchett DH, Husain M, Cherney DZ. Sodium glucose cotransporter 2 inhibitors in the treatment of diabetes mellitus: cardiovascular and kidney effects, potential mechanisms, and clinical applications. Circulation. 2016;134:752-72.

40. O'Leary DH, Polak JF, Kronmal RA, Manolio TA, Burke GL, Wolfson SK Jr, Cardiovascular Health Study Collaborative Research Group. Carotid-artery intima and media thickness as a risk factor for myocardial infarction and stroke in older adults. N Engl J Med. 1999;340:14-22.

41. Lorenz MW, Markus HS, Bots ML, Rosvall M, Sitzer M. Prediction of clinical cardiovascular events with carotid intima-media thickness: a systematic review and meta-analysis. Circulation. 2007;115:459-67.

42. Hodis HN, Mack WJ, LaBree L, Selzer RH, Liu CR, Liu $\mathrm{CH}$, et al. The role of carotid arterial intima-media thickness in predicting clinical coronary events. Ann Intern Med. 1998;128:262-9.

43. Katakami N, Yamasaki Y, Hayaishi-Okano R, Ohtoshi K, Kaneto H, Matsuhisa M, et al. Metformin or gliclazide, rather than glibenclamide, attenuate progression of carotid intima-media thickness in subjects with type 2 diabetes. Diabetologia. 2004;47:1906-13.

44. Mazzone T, Meyer PM, Feinstein SB, Davidson MH, Kondos GT, D'Agostino RB Sr, et al. Effect of pioglitazone compared with glimepiride on carotid intima-media thickness in type 2 diabetes: a randomized trial. JAMA. 2006;296:2572-81.

45. Yamasaki Y, Katakami N, Furukado S, Kitagawa K, Nagatsuka K, Kashiwagi A, et al. Long term effects of pioglitazone on carotid atherosclerosis in Japanese patients with type 2 diabetes without a recent history of macrovascular morbidity. J Atheroscler Thromb. 2010;17:1132-40.

46. Yamasaki Y, Katakami N, Hayaishi-Okano R, Matsuhisa M, Kajimoto Y, Kosugi K, et al. Alpha-glucosidase inhibitor reduces the progression of carotid intima-media thickness. Diabetes Res Clin Pract. 2005;67:204-10.

47. Mita T, Katakami N, Shiraiwa T, Yoshii H, Onuma T, Kuribayashi N, et al. Sitagliptin attenuates the progression of carotid intima-media thickening in insulin-treated patients with type 2 diabetes: the sitagliptin preventive study of intima-media thickness evaluation (SPIKE): a randomized controlled trial. Diabetes Care. 2016;39:455-64.

48. Mita T, Katakami N, Yoshii H, Onuma T, Kaneto H, Osonoi T, et al. Alogliptin, a dipeptidyl peptidase 4 inhibitor, prevents the progression of carotid atherosclerosis in patients with type 2 diabetes: the study of preventive effects of alogliptin on diabetic atherosclerosis (SPEAD-A). Diabetes Care. 2016;39:139-48.

49. Tanaka A, Murohara R, Taguchi T, Eguchi K, Suzuki M, Kitakaze M, on behalf of the PROTECT Study Investigators, et al. Rationale and design of a multicenter randomized controlled study to evaluate the preventive effect of ipragliflozin on carotid atherosclerosis: the PROTECT study. Cardiovasc Diabetol. 2016;15:133. 